\title{
Histopathology of Leishmania major infection: revisiting L. major histopathology in the ear dermis infection model
}

\author{
Silvia Dantas Cangussú ${ }^{13}$, Carolina Carvalho de Souza ${ }^{1}$, Camila França Campos ${ }^{1}$, \\ Leda Quercia Vieira², Luís Carlos Crocco Afonso33, Rosa Maria Esteves Arantes ${ }^{+} / 1$
1Departamento de Patologia Geral 2Departamento de Bioquímica e Imunologia, Instituto de Ciências Biológicas, Universidade Federal de Minas Gerais, Av. Antônio Carlos 6627, 31270-901 Belo Horizonte, MG, Brasil Departamento de Ciências Biológicas, Instituto de Ciências Exatas e Biológicas, Universidade Federal de Ouro Preto, MG, Brasil

We describe the relationship between lesion outcome and histopathological hallmarks in susceptible (BALB/c) and resistant (C57BL/6 and IL-4-deficient BALB/c) mouse strains over the course of a 12-week-infection with Leishmania major in the ear. The infiltration of mononuclear cells and polymorphonuclear cells occurred within $6 \mathrm{~h}$ and mononuclear cells predominated one week post-infection. Permissive intracellular growth of the pathogen was associated with non-healing lesions. In contrast, tissue damage and clearance of the parasite was observed in healing lesions and was associated with inducible nitric oxide synthase expression. The identification of the structural components of tissue reaction to the parasite in this study furthers our understanding of subjacent immune effector mechanisms.

Key words: Leishmania major - infectious skin diseases - pathology

Cutaneous leishmaniasis is caused by protozoan parasites of the genus Leishmania, which are transmitted as promastigotes to mammalian hosts by infected female phlebotomine sand flies. The human disease is usually self-limiting and the lesions heal spontaneously. This outcome is associated with the development of a polarised $\mathrm{T}$ helper (Th) 1 response, control of the disease and development of resistance. However, some patients develop a Th2 response and require treatment (Gaafar et al. 1995).

The inoculation of mice with Leishmania major experimentally reproduces cutaneous leishmaniasis and the development of a polarised Th1 or Th2 response results in resistance or susceptibility, respectively, to this intracellular parasite. The polarisation of the immune response depends on the strain of the infected mouse: $\mathrm{BALB} / \mathrm{c}$ mice respond to the infection with the preferential production of Th2-type cytokines, in particular IL-4 and IL-10, which are associated with disease progression and susceptibility. On the other hand, a polarised Th1 response results in macrophage activation and eradication of the parasites, allowing resistant strains, such as C57BL/6 mice, to recover from infection (Heinzel et al. 1989). Among the resistant mouse strains, there is controversy regarding the IL-4-deficient BALB/c mice (IL-4-/-). Some studies have reported that IL-4-/mice on a BALB/c background remain susceptible to $L$. major (Noben-Trauth et al. 1996), whereas others have shown that they are resistant to the parasite (Kopf et al. 1996, Mohrs et al. 1999).

Financial support: CAPES, CNPq, FAPEMIG

+ Corresponding author: rosa@icb.ufmg.br

Received 2 April 2009

Accepted 20 August 2009
Until recently, most studies examining the role of Th cells in lesions were based on the results using the classic model in which a high dose of promastigotes $\left(10^{5}-10^{7}\right)$ are subcutaneously inoculated into the footpad and/or the base of the tail (Heinzel et al. 1989, Pós et al. 2004). New protocols have since been introduced in which the inoculation of only 10-1000 parasites, the use of metacyclic promastigote preparations of $L$. major and intradermal, rather than subcutaneous, inoculation tends to better emulate the natural lesion caused by the bite of the sand fly (Belkaid et al. 2000).

In contrast to the classic high-dose model for the "natural" ear model of L. major infection there is a lack of information detailing the long-term kinetics of the structural tissue response in relation to the polarised immune response (Belkaid et al. 2000, Baldwin et al. 2007). Specifically, comparisons between susceptible $(\mathrm{BALB} / \mathrm{c})$ and resistance $(\mathrm{C} 57 \mathrm{BL} / 6)$ mouse strains have not been made, even though a role for local effector elements in parasite clearance and healing/non healing has been suggested in other studies (Belkaid et al. 2000). The accessibility to a well-demarcated lesion in the ear allows for the identification of correlations between the histopathology and other quantitative assays. We exploited this advantage of the ear model by sampling the entire lesion and studying the dynamic local histopathological changes from 6 h-12 weeks post-infection (p.i.).

Therefore, in this work a comparative study among BALB/c, C57BL/6 and the IL-4-/- BALB mouse strains examined the evolution of the size of lesions, the architectural tissue changes associated with the lesion, the inflammatory response, the mast cell (MC) density, the tissue parasite burden and inducible nitric oxide synthase (iNOS) expression over 12 weeks of infection, in order to explore the long-term histopathological pattern associated with resistance and susceptibility. 
Inbred female $\mathrm{BALB} / \mathrm{c}$ and $\mathrm{C} 57 \mathrm{BL} / 6$ mice [CEBIO, ICB/Universidade Federal de Minas Gerais (UFMG)] and male and female BALB/c mice deficient in IL-4 (IL-4-/-) (Gnotobiology Facility/ICB) were handled in accordance to international guidelines and the protocol was approved by the Animal Care Committee of UFMG (protocol 128/05). Mice were infected at 8-9 weeks of age with $10^{3}$ metacyclic L. major promastigotes, clone V1 (WHO/MHOM/80/Friedlin), intradermally into the right ear dermis, as described previously (Belkaid et al. 2000). The left ear dermis received an inoculation of 10 $\mu \mathrm{L}$ phosphate buffered saline (PBS) as a control.

BALB/c and C57BL/6 mice developed a lesion that first became clinically detectable around 3-4 weeks p.i. In contrast, IL-4-/- mice did not develop a significant lesion at the injection site (Fig. 1A). The growth of the lesion was assessed by measuring the thickness of the ear and was similar in all lineages until four weeks p.i., after which $\mathrm{BALB} / \mathrm{c}$ mice presented larger lesions than the C57BL/6 mice. The BALB/c mice developed severe lesions at the site of cutaneous inoculation and exhibited a progressive increase, with ulceration, necrosis and extensive inflammatory reaction with no healing activity until 12 weeks p.i (Fig. 1B). In contrast, in the C57BL/6 mice the lesion size peaked at seven weeks p.i and healed between 8-12 weeks, as previously described (Belkaid et al. 2000, Baldwin et al. 2007). Occasionally, this resulted in a measurable induration $(<1 \mathrm{~mm})$ (Fig. 1C). The lesions of $\mathrm{BALB} / \mathrm{c}$ and $\mathrm{C} 57 \mathrm{BL} / 6$ mice were significantly larger than the lesions of IL-4-/- mice, which showed very small variations in lesion size and histology, when present (Fig. 1D). Our results are similar to observations from other authors in BALB/c mice deficient in the IL-4 receptor $\alpha$-chain (IL-4R $\alpha-/-$ ) using the classic model (Kopf et al. 1996, Mohrs et al. 1999). The only previous study in IL-4-/mice on the C57BL/6 background using the natural model also demonstrated that the dermal lesions in these mice resolve by week 10 (Belkaid et al. 2000).

The presence of parasites at the site of infection was confirmed by the immunostaining of paraffin sections for L. major. The labelled streptavidin-biotin (LSAB) method was used in conjunction with a rabbit anti-serum against $L$. major described previously (Tafuri et al. 2004). The immunolabeled area in the paraffin sections was automatically measured. At the early time points (until 1 week), parasites were not detected in any mouse lineage. Parasites were initially detected at 4.5 weeks in all lineages, but significant differences between the lineages were not detected until week $10(p=0.023)$ and week 12 $(\mathrm{p}=0.003)$. Previous studies using infection with a low dose of L. major in C57BL/6 mice also failed to detect any change in parasite load in the dermis, even using limiting dilution evaluation during the first four weeks of infection (Belkaid et al. 2000). In BALB/c mice the higher density of parasites, represented by an extensive number of vacuolated macrophages containing a large number of parasites in the lesion site (Fig. 2G, inset), occurred at 12 weeks p.i. and corresponded to the larger lesions. However, in C57BL/6 and IL-4-/- mice the parasite density did not vary from 4.5-12 weeks, indicating a steady state parasite burden. A significant smaller la-
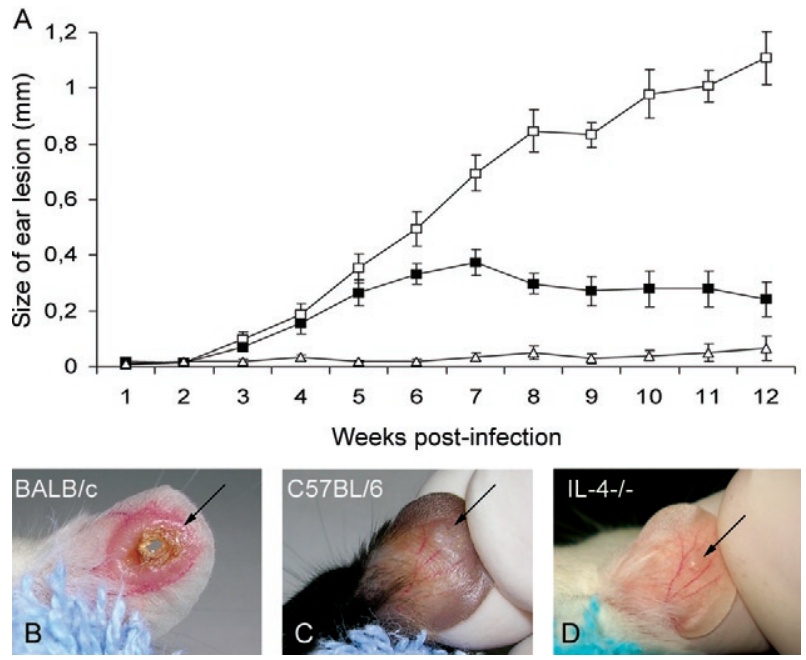

Fig. 1: time course of experimental cutaneous leishmaniasis in susceptible (BALB/c) (口), resistance (C57BL/6) (ロ) and IL-4-/- ( $\Delta$ ) mice. Differences in lesion size between BALB/c and C57BL/6 mice were significant $(\mathrm{p}<0.001)$ from week 8 onward, whereas differences in lesion sizes between BALB/c and IL-4-/- $(\mathrm{p}<0.001)$ and between C57BL/6 and IL-4-/- $(\mathrm{p}<0.05)$ were significant from week 5 onward $(\mathrm{n}=5-24$ mice per group) (A). Macroscopic aspect of the lesions (12 wks p.i) in BALB/c (B), C57BL/6 (C) and IL-4-/- (D) mice.

belled parasite area $/ \mathrm{mm}^{2}$ was detected in IL-4-/- lesions at 12 weeks p.i $(4.01 \pm 1.13)$ compared to $\mathrm{BALB} / \mathrm{c}$ mice $(69.2 \pm 15.9, \mathrm{p}=0.027)$, but not to $\mathrm{C} 57 \mathrm{BL} / 6(10.07 \pm 4.37$, $\mathrm{p}=0.272$ ) (Fig. 2G, I, insets).

Controversially, the susceptibility of IL-4-/- or IL4R $\alpha-/-$ BALB/c mice infected with higher dose of $L$. major in subcutaneous sites has been attributed to differences in the parasite substrain, the genetic origin of the embryonic stem cells used in the generation of the knockout mouse, the number of backcrosses and on minor variations in the number of parasites used to infect the mice (Kropf et al. 2003). However, our results reinforce observations that IL-4 deficiency confers resistance to L. major infection in otherwise BALB/c.

The morphometrical determination of the inflammatory cell density in the dermis (cells/ $\mathrm{mm}^{2}$ ) (Table) was based on the classic morphological pattern of mononuclear cells, neutrophils, eosinophils and MCs. The cell composition of lesions observed in the ears of L. majorinfected BALB/c and C57BL/6 mice did not differ (Table). Lesions from IL-4 -/- mice were very small, did not expand over the 12-week-course and were not included in the morphometric analysis. However, these lesions did present with infrequent, predominantly mononuclear cells in the dermis and were associated with relatively intense vascular reactivity that was microscopically visible from 24 h-12 weeks p.i (Fig. 2C, F, I). Previous studies of C57BL/6 mice infected with low-dose L. major (Belkaid et al. 2000) did not describe significant changes in the cellularity of the dermis during the first four weeks p.i., but the quantification was done on cells isolated from epidermal preparations. The digestion procedure necessary for obtaining a dermal cell suspension may 
prevent the adequate analysis of small and scarcely populated lesions at the very initial time points. Since the initial injury is small and not easily detected by the naked eye, careful sampling is required, preferentially under a stereomicroscope, in order to detect the very early inflammatory changes induced by the low dose inoculum, as shown here. Although we succeeded in sampling the lesion at very early time points, major differences in the cell types and tissue reaction were not detected in the different mouse strains throughout the experiment, despite the consistent susceptibility or resistance patterns indicated by the lesion size and aspect. Despite the absence of differences among strains in this initial period, our morphometric analysis (Table) clearly shows that cell migration and accumulation into the inoculation site increases progressively and changes from mixed leukocytes to predominantly mononuclear cells from $6 \mathrm{~h}-4.5$ weeks, when the lesion is already macroscopically visible and lineages begin to develop different sized lesions. A clear-cut difference appeared in the lesions in both lineages one week after inoculation. The predominant cells observed at this time point and onwards, were mononuclear leukocytes, but polymorphonuclear leukocytes (eosinophils and neutrophils) were still present in both lineages (Table), in agreement with another study (Beil et al. 1992) that determined that polymorphonuclear cells and mononuclear cells accounted for $50 \%$ of the cells in C57BL/6 lesions. However, in this same study polymorphonuclear cells predominated in BALB/c lesions even though the mice were inoculated with a highdose inoculum and only small samples were examined using electron microscopy.

Four and a half weeks p.i., mononuclear cells constituted the major population in the ear dermis of both $\mathrm{C} 57 \mathrm{BL} / 6$ and $\mathrm{BALB} / \mathrm{c}$ mice. Lesions from C57BL/6 mice (Fig. 2B, E, H) appeared less organised because of cell debris between the effector cells. Areas of necrosis, in which free amastigote parasites could be seen among inflammatory cells, were evident. From the beginning there was intense tissue reactivity with edema, dilated vessels and a reactive endothelium. Occasionally, granulocyte infiltration was observed, resulting in micro-abscesses. At 12 weeks p.i, inflammatory infiltration and cell debris was scarce in C57/BL6 mice and fibroblast proliferation and collagen formation appeared throughout the lesion. The lesions in IL-4-/- mice presented with a discrete inflammatory infiltrate that was predominantly mononuclear cells and did not change significantly over time.

Several innate immune mediators, including those released by MC, play an important role in the outcome of the disease. We examined the role of MC in the susceptibility and resistance of both C57BL/6 and BALB/c mice to experimental cutaneous infection with a low dose of L. major. MCs were identified histologically following the staining of paraffin sections with $1 \%$ toluidine blue in phosphate buffer, $\mathrm{pH}$ 1.5. Images of the sections were automatically analysed and the density $\left(\mathrm{MC}\right.$ per $\left.\mathrm{mm}^{2}\right)$ and absolute number of MC per lesion was determined.

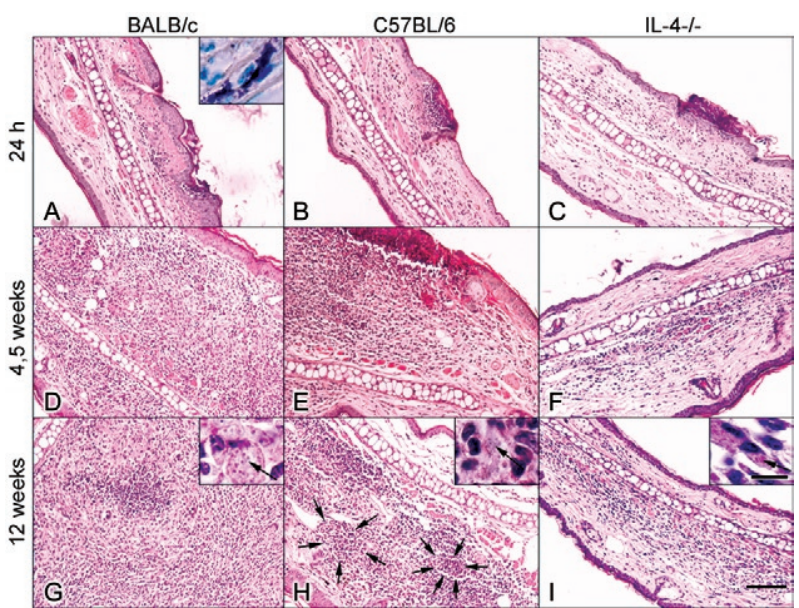

Fig. 2: comparative microscopic features of ear lesions along the experimental period. Lesions of susceptible BALB/c (A, D, G), resistance C57BL/6 (B, E, H) and IL-4-/- (C, F, I.) mice documented at 24 h (A-C), 4,5 weeks (D-F) and 12 weeks (G-I) post-infection. Arrows in $\mathrm{H}$ indicate micro abscesses. At 12 weeks, extensive collection of vacuolated and heavily parasited macrophages in BALB/c (G, inset) and weakly parasited macrophages in C57BL/6 (H, inset) and IL-4-/(I, inset). Bar (A-I) $=100 \mu \mathrm{m}$. Bar (insets) $=10 \mu \mathrm{m}$.

There was no significant difference in $\mathrm{MC}$ density at any time point between the C57BL/6-BALB/c mice (Table), in agreement with results obtained by Wershil et al. (1994). However, the absolute number of MCs per lesion increased significantly in BALB/c compared to C57BL/6 mice $(\mathrm{p}<0.001)$ at 10 and 12 weeks p.i. (Table). The absolute number of MCs outside the lesion and in PBSinjected control ears did not differ significantly between the lineages throughout the 12 weeks of examination (data not shown). We detected extensive degranulation of cutaneous MCs in both C57BL/6 and BALB/c mice, as well as in the small lesions of IL 4-/- mice, especially immediately following inoculation (6-24 h p.i), (Fig. 2A, inset). MC degranulation before challenge with L. major makes BALB/c more resistant to infection (Romão et al. 2009). Additionally, previous research has suggested that MCs may participate in the exacerbation of the immune response (Pós et al. 2004).

The antimicrobial activity of nitric oxide $(\mathrm{NO})$ in vivo has been previously demonstrated in C57BL/6 mice and other resistant strains infected with L. major (Stenger et al. 1996, Belkaid et al. 2000, Chakour et al. 2003) and in human cutaneous leishmaniasis by immunohistochemistry (Qadoumi et al. 2002). Importantly, the increased tissue expression of iNOS in the C57BL/6 strain, relative to the BALB/c strain, has been closely associated with resistance to L. major (Diefenbach et al. 1998) in both the classic and natural models (Belkaid et al. 2000) of infection. The persistence of Leishmania is thought to be required for the maintenance of host immunity, while the simultaneous expression of iNOS prevents uncontrolled parasite replication and reduces the level of $\mathrm{T}$ cell activity (Stenger et al. 1996, Chakour et al. 2003), suggesting a correlation between iNOS expression and disease outcome. 
TABLE

Number and types of cells of dermis lesion post inoculation of $10^{3}$ Leishmania major metacyclicis in susceptible (BALB/c) and resistance $(\mathrm{C} 57 \mathrm{BL} / 6)$ mice

\begin{tabular}{lcccccc}
\hline & \multicolumn{3}{c}{ C57BL/6 } & \multicolumn{3}{c}{ BALB/c } \\
\cline { 2 - 7 } Cell types & $6 \mathrm{~h}$ & 4.5 weeks & 12 weeks & $6 \mathrm{~h}$ & 4.5 weeks & 12 weeks \\
\hline Mononuclear $/ \mathrm{mm}^{2}$ & $1.25 \pm 0.39$ & $3.48 \pm 0.31$ & $3.17 \pm 0.22$ & $1.13 \pm 0.18$ & $4.91 \pm 0.18$ & $3.24 \pm 0.15$ \\
Polimorphonuclear neutrophils $/ \mathrm{mm}^{2}$ & $1.62 \pm 0.45$ & $0.17 \pm 0.08$ & $0.37 \pm 0.09$ & $1.39 \pm 0.21$ & $0.25 \pm 0.09$ & $1.02 \pm 0.52$ \\
Polimorphonuclear eosinophils $/ \mathrm{mm}^{2}$ & $0.03 \pm 0.03$ & $0.35 \pm 0.17$ & $0.65 \pm 0.24$ & $0.04 \pm 0.04$ & $0.97 \pm 0.30$ & $0.82 \pm 0.32$ \\
\hline & 4.5 weeks & 10 weeks & 12 weeks & 4.5 weeks & 10 weeks & 12 weeks \\
\hline Mast cell $/ \mathrm{mm}^{2}$ & $7.93 \pm 1.05$ & $5.8 \pm 1.23$ & $8.14 \pm 2.09$ & $7.40 \pm 2.11$ & $4.67 \pm 0.59$ & $5.48 \pm 0.67$ \\
Absolute number of mast cells & $68 \pm 13.7$ & $86.8 \pm 21.5$ & $164.8 \pm 19.7$ & $56.8 \pm 15.6$ & $274.2 \pm 7.5^{a}$ & $357.1 \pm 60.9^{a}$ \\
\hline
\end{tabular}

$a$ : values are significantly greater than the other group at 10 and 12 weeks $(\mathrm{p}<0.001)$.

We used immunohistological techniques to characterise the cell distribution and quantification of iNOS expression in C57BL/6, BALB/c and IL-4-/- mice following intradermal inoculation of $L$. major into the ear. The LSAB method was used on 4- $\mu \mathrm{m}$-thick paraffin sections of ear using a rabbit polyclonal antibody against iNOS (1:10.000; Chemicon, Temecula, CA). The immunolabeled area was measured and the results are expressed as the iNOS labelled area $/ \mathrm{mm}^{2}$. This study provides the first documentation of the in situ antimicrobial function of NO in the L. major natural model. The expression of iNOS protein in the lesions of BALB/c was low throughout the course of infection, whereas the expression in C57BL/6 and IL-4 -/- mice was very low between 1-4.5 weeks p.i., but significantly increased after 12 weeks p.i $(\mathrm{p}<0.01)$. Also, iNOS expression was significantly increased in $\mathrm{C} 57 \mathrm{BL} / 6$ mice when compared to $\mathrm{BALB} / \mathrm{c}$ at 12 weeks p.i ( $p=0.0021)$ (Fig. 3A-D). Moreover, only a few parasites were detected in the iNOS-immunopositive areas, whereas a large number of parasites was found in iNOS-negative regions, independent of the lineage.

Our results confirmed preliminary studies in C57BL/6 and $\mathrm{BALB} / \mathrm{c}$ mice infected with a high dose of L. major (Stenger et al. 1996) and also confirmed the resistant phenotype of IL-4 -/- mice on a BALB/c background (Kopf et al. 1996, Mohrs et al. 1999). This study reinforces the role of local iNOS expression as a marker of the resistant phenotype. Recent progress in pathogenesis research has significantly contributed to a better understanding of protective immunity against $L$. major and demonstrates the finely tuned host-parasite relationship. Revisiting the components of the local inflammatory reaction is particularly important in the context of novel therapeutic approaches that focus on cell-based therapies or manipulates the local infection site.

\section{ACKNOWLEDGEMENTS}

To Mirna M d'Auriol-Souza, for preparing the tissue samples, to Juan P Macedo, for parasites, and to Lucélia Pinheiro, Maria M Figueiredo and Antonio M Vaz, for animal care.

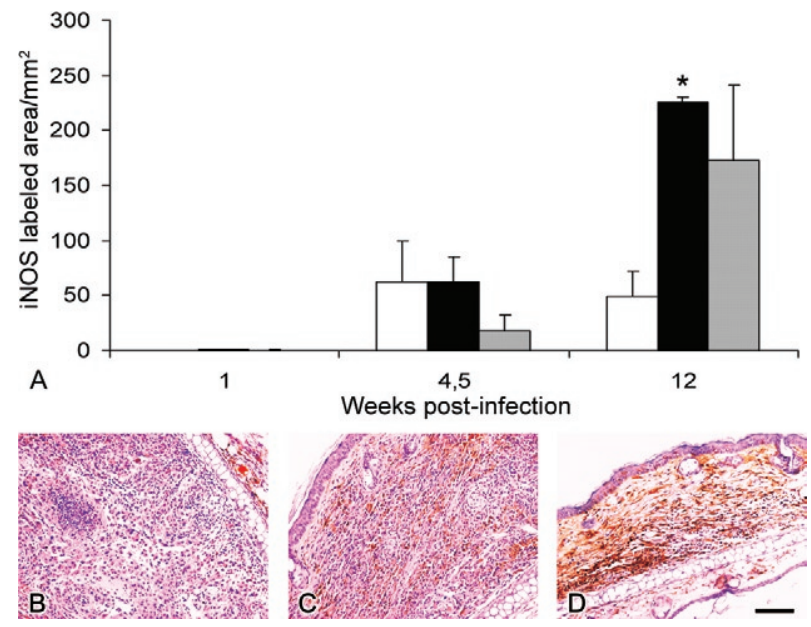

Fig. 3: oxide synthase (iNOS) expression immunochemistry and quantification. The iNOS labeled area/mm $\mathrm{mm}^{2}$ in susceptible BALB/c (white bar), resistance C57BL/6 (black bar) and IL-4-/- (gray bar) mice following intradermal inoculation of $10^{3}$ L. major metacyclics (A). Asterisks mean statistically significant difference between BALB/c and C57BL $/ 6$ ( $p<0.001)(n=3-4$ mice per group). Increased iNOS expression was detected by 12 weeks post-infection in lesions of C57BL/6 (C) or IL-4-/- (D) mice, but not in BALB/c (A). Bar $=100 \mu \mathrm{m}$.

\section{REFERENCES}

Baldwin T, Sakthianandeswaren A, Curtis JM, Kumar B, Smyth GK, Foote SJ, Handman E 2007. Wound healing response is a major contributor to the severity of cutaneous leishmaniasis in the ear model of infection. Parasite Immunol 29: 501-513.

Beil WJ, Meinardus-Hager G, Neugebauer DC, Sorg C 1992. Differences in the onset of the inflammatory response to cutaneous leishmaniasis in resistant and susceptible mice. J Leukoc Biol 52: 135-142.

Belkaid Y, Mendez S, Lira R, Kadambi N, Milon G, Sacks D 2000. A natural model of Leishmania major infection reveals a prolonged "silent" phase of parasite amplification in the skin before the onset of lesion formation and immunity. J Immunol 165: 969-977.

Chakour R, Guler R, Bugnon M, Allenbanch C, Garcia I, Mauël J, 
Louis J, Tacchini-Cottier F 2003. Both the Fas ligand and inducible nitric oxide synthase are needed for control of parasite replication within lesions in mice infected with Leishmania major whereas the contribution of tumor necrosis factor is minimal. Infect Immun 71: 5287-5295.

Diefenbach A, Schindler H, Donhauser N, Lorenz E, Laskay T, MacMicking J, Rollinghoff M, Gresser I, Bogdan C 1998. Type 1 interferon (IFNalpha/beta) and type 2 nitric oxide synthase regulate the innate immune response to a protozoan parasite. Immunity 8: 77-87.

Gaafar A, el Kadaro AY, Theander TG, Permin H, Ismail A, Kharazmi A, el Hassan AM 1995. The pathology of cutaneous leishmaniasis due to Leishmania major in Sudan. Am J Trop Med Hyg 52: $438-442$.

Heinzel FP, Sadick MD, Holaday BJ, Coffman RL, Locksley RM 1989. Reciprocal expression of interferon gamma or interleukin 4 during the resolution or progression of murine leishmaniasis. Evidence for expansion of distinct helper T cell subsets. $J$ Exp Med 169: 59-72.

Kopf M, Brombacher F, Kohler G, Kienzle G, Widmann KH, Lefrang K, Humborg C, Ledermann B, Solbach W 1996. IL-4-deficient Balb/c mice resist infection with Leishmania major. J Exp Med 184: 1127-1136.

Kropf P, Herath S, Weber V, Modolell M, Muller I 2003. Factors influencing Leishmania major infection in IL-4-deficient BALB/c mice. Parasite Immunol 25: 439-447.

Mohrs M, Ledermann B, Kohler G, Dorfmuller A, Gessner A, Brombacher F 1999. Differences between IL-4- and IL-4 recep- tor alpha-deficient mice in chronic leishmaniasis reveal a protective role for IL-13 receptor signaling. J Immunol 162: 7302-7308.

Noben-Trauth N, Kropf P, Muller I 1996. Susceptibility to Leishmania major infection in interleukin-4-deficient mice. Science 271: 967-990.

Pós Z, Müller K, Novák I, Buzás E, Solbach W, Falus A, Laskay T 2004. Different patterns of the L-histidine decarboxylase ( $h d c$ ) gene expression in mice resistant and susceptible to experimental cutaneous leishmaniasis. Inflamm Res 53: 38-43.

Qadoumi M, Becker I, Donhauser N, Rollinghoff M, Bogdan C 2002. Expression of inducible nitric oxide synthase in skin lesions of patients with American cutaneous leishmaniasis. Infect Immun 70: 4638-4642.

Romão PRT, Santiago HC, Ramos CDL, De Oliveira CFE, Monteiro MC, Cunha FQ, Vieira LQ 2009. Mast cell degranulation contributes to susceptibility to Leishmania major. Parasite Immunol 31: $140-146$.

Stenger S, Donhauser N, Thuring H, Rollinghoff M, Bogdan C 1996. Reactivation of latent leishmaniasis by inhibition of inducible nitric oxide synthase. J Exp Med 183: 1501-1514.

Tafuri WL, Santos RL, Arantes RM, Gonçalves R, de Melo MN, Michalick MS, Tafuri WL 2004. An alternative immunohistochemical method for detecting Leishmania amastigotes in paraffin-embedded canine tissues. J Immunol Methods 292: 17-23.

Wershil BK, Theodos CM, Galli SJ, Titus RG 1994. Mast cells augment lesion size and persistence during experimental Leishmania major infection in the mouse. J Immunol 152: 4563-4571. 